\title{
FORMACIÓN BRUTA DE CAPITAL EN BIENES DE EQUIPO EN CHILE, 1856-1930. FUENTES NACIONALES Y EXTRANJERAS
}

\author{
GROSS CAPITAL FORMATION \\ IN EQUIPMENT IN CHILE, 1856-1930. \\ NATIONAL AND FOREIGN SOURCES
}

\author{
Cristián Ducoing \\ Universitat Pompeu Fabra, Barcelona, España, <cristian.ducoing@upf.edu> \\ Xavier Tafunell \\ Universitat Pompeu Fabra, Barcelona, España, <xavier.tafunell@upf.edu>
}

\begin{abstract}
Resumen. Pese a contar con unas de las mejores estadísticas de los países en desarrollo, existen lagunas en las series económicas de Chile, las cuales se han corregido a lo largo de los años. El trabajo conjunto de los autores ha permitido conocer las similitudes y diferencias entre una evaluación basada en las estadísticas chilenas y otra elaborada con las estadísticas del comercio exterior del G-3 (Alemania, Estados Unidos y Gran Bretaña). El objetivo del artículo es contrastar las series de inversión en maquinaria para Chile desde 1856 hasta 1930 y servir de base para otros estudios que quieran aportar nuevas series que permitan entender el desarrollo de Chile en el largo plazo.
\end{abstract}

Palabras clave: comercio internacional, inversión en maquinaria, estadísticas históricas.

Abstract. Despite having the best statistics in developing countries, there are a number of gaps in Chile's economic series, which have been being corrected over the years. Our joint work has allowed knowing the similarities and differences between series made from the Chilean statistical yearbooks and official trade statistics of G-3 (Germany, USA and Great Britain). The article aims to contrast both machinery investment series for Chile from 1856 to 1930 and provide a basis for other studies that want to make new series for understanding the development of Chile in the long term.

Key words: international commerce, machinery investment, historical statistics.

Fecha de recepción: octubre de 2011. Fecha de aceptación: febrero de 2012.

Am. Lat. Hist. Econ., año 20, núm. 1, enero-abril, 2013, pp. 5-34 


\section{INTRODUCCIÓN}

$\mathrm{E}$ estudio de la evolución macroeconómica de las naciones latinoamericanas se enfrenta, en muchas ocasiones, a una escasez de oferta de fuentes estadísticas. Esto ocurre rara vez -o bien, la escasez no reviste especial gravedad- en la era propiamente estadística, entendiendo por tal aquella en la cual instituciones oficiales elaboran las cuentas nacionales. Como se sabe, la era estadística se inició alrededor de 1950 en la mayor parte de América Latina. Para periodos anteriores a ese momento histórico el investigador suele disponer de pocas publicaciones estadísticas que den cuenta de la dinámica macroeconómica y la situación estructural de las economías de la región: los censos -agrícolas, industriales e incluso de la población- son muy contados, las más de las veces inexistentes; las estadísticas fiscales suelen ser discontinuas y fragmentarias, y las actividades productivas generalmente no han quedado registradas en ninguna fuente accesible. Es verdad, apresurémonos a señalarlo, que Chile no encaja en este diagnóstico: las fuentes estadísticas son de una gran riqueza, lo que brinda a los analistas de la historia económica chilena un potencial de conocimiento excepcional en el contexto latinoamericano. Pero, si apuntamos a las posibilidades que están al alcance de los historiadores económicos de América Latina en general, habrá que convenir que el diagnóstico no es errado. Dentro de este panorama un tanto desolador las estadísticas oficiales del comercio exterior (en adelante ECE) aparecen como una fuente estadística fundamental, hasta el punto que para muchos investigadores se han convertido en un instrumento de conocimiento imprescindible e insustituible. El papel desempeñado por el comercio exterior como motor del crecimiento de unas economías abocadas a los mercados internacionales, hasta la gran depresión de los años treinta, ha dado todavía mayor centralidad a las ECE como fuente de base de las interpretaciones formuladas por los historiadores económicos. Sin embargo, estos no han sometido a prueba la fiabilidad de la fuente, la han utilizado sin saber cuáles eran los márgenes de error de los datos, o bien, en el peor de los casos, han usado de manera muy contenida las ECE -podríamos decir que ha habido represión documental-, ante la sospecha que la fuente adolecía de graves deficiencias. Es urgente e importante, pues, realizar algunos ejercicios de contraste sistemáticos de las ECE con el propósito de determinar su grado de fiabilidad.

El presente artículo persigue este objetivo. Hasta cierto punto, hemos seguido la senda trazada por otros autores. Los trabajos seminales de Federico y Tena midieron el grado de fiabilidad de las ECE de una amplia muestra de países desarrollados y en vías de desarrollo, a partir de las 
magnitudes globales de los flujos comerciales bilaterales. ${ }^{1}$ Recientemente, ambos autores han profundizado en esta línea de análisis sin abandonar el enfoque global, centrándolo en naciones latinoamericanas. ${ }^{2}$ Carreras y Badía-Miró, y también Folchi y Rubio han evaluado con precisión los márgenes de discrepancia entre los datos de las ECE de los países latinoamericanos y de sus principales socios comerciales, en lo que se refiere al comercio del carbón como producto específico en un año determinado, o bien, en un periodo no muy largo. ${ }^{3}$ Nuestro propósito va más allá de los estudios anteriores porque abarca un amplio y complejo grupo de bienes y porque el contraste se lleva a cabo de manera sistemática, para un dilatado periodo. En concreto, el presente artículo puede ser descrito como un ejercicio de verificación de la calidad de la ECE chilena a través de la confrontación entre una elaboración estadística realizada a partir de los datos que aquella contiene sobre importaciones de bienes de equipo entre 1856 y 1930 y otra efectuada de forma independiente y basada en los datos sobre exportación del mismo tipo de bienes que figuran en las ECE de los principales países proveedores de esos bienes. La evidencia empírica que se pone en juego en el ejercicio -la maquinaria y otros equipos- es de suma trascendencia para la comprensión del proceso de crecimiento de la economía chilena. Pese a ello, en este ejercicio dicha evidencia tiene un carácter puramente instrumental. Dejaremos totalmente de lado el análisis de la evolución de la inversión (virtualmente equivalente a la importación de bienes de equipo), para centrarnos en exclusiva en las similitudes y disimilitudes entre las estimaciones de la misma basadas en las ECE de Chile y de otros países. El artículo se estructura en seis apartados además de esta introducción. El siguiente apartado está dedicado a la descripción crítica de la fuente chilena. El tercero se ocupa de las fuentes de los principales socios comerciales de Chile, es decir, Alemania, Bélgica, Estados Unidos y Reino Unido. El cuarto compara las estimaciones obtenidas de ambas fuentes, examinando desde distintos ángulos el grado de afinidad de las series. En el quinto formulamos hipótesis explicativas de las discrepancias más significativas. El artículo cierra con un conciso apartado de conclusiones. Avancemos el resultado del balance: la ECE chilena muestra una gran consistencia a lo largo de todo el periodo estudiado, de lo que cabe concluir que puede ser manejada con confianza como fuente para múltiples aproximaciones cuantitativas sobre el desenvolvimiento de la economía nacional en el largo siglo posterior a la independencia.

\footnotetext{
${ }^{1}$ Federico y Tena, “Accuracy”, 1991, y Tena, "Estadísticas”, 1992.

2 Tena y Federico, "What?", 2011.

${ }^{3}$ Carreras y Badía-Miró, "Fiabilidad", 2008, y Folchi y Rubio, "Consumo”, 2008.
} 


\section{LAS FUENTES OFICIALES CHILENAS}

El proceso de construcción de una serie de maquinaria y otros equipos para el periodo de 1856-1930 se ha llevado a cabo con el uso de diversas fuentes oficiales chilenas. ${ }^{4}$ Considerando que el principal input de la investigación son las fuentes de la Estadística comercial de Chile, es perentorio hacer una valoración sobre la disponibilidad, estado y conservación de las mismas.

Desde que se creó la Oficina de Estadísticas en Chile se ha mantenido un estándar apreciado internacionalmente en la recopilación de datos socioeconómicos. ${ }^{5}$ No obstante, encontrar los anuarios de comercio exterior de Chile no es una tarea del todo fácil. No todos los anuarios están en condición física en la Biblioteca Nacional y al menos todo el periodo de 1860-1870 ${ }^{6}$ ha desaparecido de las microfichas. Otro grupo de anuarios de comercio exterior de Chile se encuentra en el Instituto Nacional de Estadísticas, pero muchos en condición deplorable y varios volúmenes han desaparecido, por ejemplo, la década de 1870. Hace pocos años, el Instituto Nacional de Estadísticas llevó a cabo una digitalización de los anuarios estadísticos de la República de Chile, pero una revisión minuciosa de la digitalización muestra que en gran parte de los años no se encuentran los datos de comercio exterior, lo que hace inútiles estos archivos digitales para presentarlos en este artículo.

\footnotetext{
${ }^{4}$ La elaboración de series de formación bruta de capital en maquinaria para Chile en el periodo de 1830 a 1938 ha contado con diversas aportaciones en el pasado. Han existido ciertas intenciones de conocer el proceso de modernización de la economía chilena desde el punto de vista de la inversión en maquinaria, especialmente desde que se iniciaron los estudios sobre la industrialización chilena y sus características esenciales en comparación con los procesos de industrialización ocurridos en Europa y América Latina. Entre las series construidas, destacan las realizadas por Carmagnani, Sviluppo, 1971, y Kirsch, Industrial, 1977. Aunque aportan valores agregados útiles para el estudio de la economía chilena en el largo plazo, ambas cometen el error de sobrestimar la importación de capital en el periodo de 1870 a 1890, al contabilizar los sacos utilizados para la actividad salitrera y triguera. En defensa de Carmagnani se debe argumentar que su intención fue relacionar el proceso de industrialización chileno con un fallido proceso de crecimiento sostenido, donde la formación bruta de capital en maquinaria desempeña un papel importante, pero no el fundamental, por tanto, que no haya habido una específica preocupación por la calidad de la reconstrucción de la serie de maquinaria es absolutamente entendible, ya que antes de la formación de capital, al autor en cuestión le interesaba reconstruir el alcance y la importancia que tuvieron las manufacturas en la economía chilena. En el caso de Kirsch, su análisis se centra en la participación de la maquinaria destinada específicamente a la industria.

${ }^{5}$ Según Mamalakis, Chile es un país privilegiado para estudiar el subdesarrollo, gracias a sus ricas fuentes estadísticas, véase Reynolds y Mamalakis, Essays, 1965, p. 13.

${ }^{6}$ Por ejemplo, los datos referentes al comercio exterior de 1872 y 1873 tienen varias hojas desaparecidas (lamentablemente, entre ellas las de maquinaria).

${ }^{7}$ La mayoría de los anuarios estadísticos digitalizados no contienen los datos de comercio exterior, especialmente el periodo de 1850 a 1912. En años más cercanos al presente, estos datos sí se encuentran.
} 
En lo que respecta al análisis monetario, entre 1844 y 1925, las monedas con las cuales se presentaban los artículos de importación y exportación eran los pesos oro de $38 \mathrm{~d}$ y $18 \mathrm{~d}$, o pesos de 18 peniques. Esta denominación será reemplazada por los pesos oro de $6 \mathrm{~d}$ o pesos de 6 peniques, ${ }^{8}$ al crearse el Banco Central de Chile en 1925 y promover el uso de billetes y nuevos medios de pagos.

Aclarada la situación en la que se encuentran las fuentes principales, se describe cómo se compone esta fuente. Como se había mencionado con anterioridad, en primer lugar determinaremos la importación en maquinaria por sectores según su aparición en la estadística. Como toda forma de recopilación de datos, esta ha ido cambiando a lo largo del tiempo, lo que dificulta su recopilación. Es posible distinguir cinco formas de presentación de las estadísticas de maquinaria según los periodos en que estas aparecieron o fueron modificadas respecto a sus precedentes: ${ }^{9} a$ ) en el periodo de 1844 a $1860^{10}$ no hay una definición clara de categorías específicas; los artículos importados están ordenados alfabéticamente y en ciertas ocasiones, además de alfabéticamente, por puerto de entrada $;^{11}$ b) de 1861 a $1889^{12}$ aparece la primera categorización de las maquinarias y herramientas como un rubro separado, junto con una categoría dedicada a los transportes. Estas dos categorías son sumamente amplias y requieren de una exhaustiva revisión año con año. En primer lugar aparecen en la categoría "maquinarias, equipos y artefactos para las industrias" que agrupa toda la maquinaria, instrumentos y utensilios que utilizan las industrias agrícolas, mineras y la industria propiamente. Esta situación genera grandes confusiones y es lo que lleva a pensar en cierta sobrestimación de las series pasadas. ${ }^{13}$ Principalmente porque entre 1881 y 1909 y debido a la excepcional actividad salitrera, se contabilizan los sacos dentro de la categoría "maquinaria y útiles". Por ejemplo, en 1888 los sacos suman 1617812 pesos de $18 \mathrm{~d}$ que equivale a $27.1 \%$ de la importación total de

${ }^{8}$ La denominación pesos oro de $38 \mathrm{~d}, 18 \mathrm{~d}$ o $6 \mathrm{~d}$ responde a la denominación de la estadística chilena a la moneda nacional y su cambio estimado con respecto a las divisas (especialmente la libra esterlina).

${ }^{9}$ Para tener una historia detallada de la historia de la estadística económica de Chile en el periodo estudiado en este artículo, véanse Censo, 1939, e Instituto Nacional de Estadísticas, Chile, en $\langle$ http://www.ine.cl/canales/corporativo/nuestra_institucion/historia/historia.php $>$.

${ }^{10} \mathrm{El}$ recuento se inicia desde 1844 porque, previo a esto, no existe un recuento ordenado de las importaciones de maquinaria por parte del gobierno chileno.

${ }^{11}$ Esto dificulta la agregación, pero en el futuro permitiría un estudio sobre la inversión regional y las diferencias de producto en las zonas chilenas.

${ }^{12}$ En diversos años, por ejemplo en 1870 y 1871, la separación de artículos sigue siendo por orden alfabético, y sólo al principio se encuentra un resumen que explica por agrupaciones de artículos cuál es la participación de la maquinaria en el total de las importaciones.

${ }^{13}$ Véanse Lagos, Industria, 1966; Carmagnani, Sviluppo, 1971, y Kirsch, Industrial, 1977. 
"maquinaria" de ese año. ${ }^{14}$ En un rubro aparte se encuentra "locomoción, ferrocarriles y telégrafos", categorización que tampoco es absolutamente referida a los equipos de transporte manufacturados, ya que se incluyen los caballos y los burros; c) en 1903 las referencias se trasladan a un solo rubro con subsectores. ${ }^{15}$ El principal es "máquinas, instrumentos, herramientas, aparatos y sus piezas"; los subsectores son denominados "artes y ciencias", "minería", "agricultura", "industrias fabriles" y "locomoción". Esta clasificación desaparece en 1914; d) finalmente, desde 1914 y hasta 1930 la forma de agrupar maquinaria y equipos se dividió en dos rubros principales denominados "maquinarias, útiles y herramientas para las industrias" y "materiales y útiles para la locomoción”. El primero se integra de los subsectores "maquinarias, útiles y herramientas para la minería", "maquinarias, útiles y herramientas para la agricultura”, "máquinas, útiles y herramientas para las industrias no especificadas", "motores, calderas y sus repuestos" y "aparatos y material eléctrico". El segundo rubro principal se integra de materiales y útiles para los ferrocarriles y tranvías, materiales y útiles para la navegación y vehículos no especificados y sus repuestos. ${ }^{16}$

Para desagregar los datos, se dispone de las características de la maquinaria importada que otorga la Estadística comercial de Chile, la cual distingue, dependiendo del año, la maquinaria para agricultura, minería, industria, artes y ciencias, servicios, y transporte y equipos (véase cuadro 1).

El cuadro 1 presenta la forma de agregación de los distintos activos recopilados por medio de los anuarios de comercio exterior de la república de Chile. Se ha mencionado que en este extenso periodo, las categorizaciones y los tipos de maquinaria han variado. En este sentido, se llevaron a cabo ciertas modificaciones en la agregación de los datos, que en la medida que van avanzando los años, el sector "servicios y ciencias", que reúne lo que la Estadística comercial de Chile considera instrumentos para ciencias,

${ }^{14}$ Esta situación se repite a lo largo de la década de 1880, se sigue insertando los sacos en la contabilidad de maquinaria. En 1899 ya desaparece la contabilidad de los sacos en el rubro de maquinaria y equipos. Esto cambia de forma absoluta la contabilidad porque sin ir muchos años atrás, en 1897, el valor de los sacos alcanzó los 5148841 pesos, siendo que ese año se importaron "maquinarias por un valor de 20088890 pesos, significando un cuarto de la formación bruta de capital en maquinaria. Véase Estadística, 1898.

${ }^{15}$ Previamente, desde 1878 , existe un resumen del comercio exterior al final de cada volumen, donde se indican las "maquinarias, útiles y herramientas para las industrias" y en otro apartado "locomoción y otros vehículos" o simplemente locomoción. El problema de estos apartados, especialmente en el caso la maquinaria, es que agrega todas las máquinas en el mismo rubro (maquinaria para las industrias), refiriéndose a todos los sectores productivos.

${ }^{16}$ Respecto a los automóviles, un problema que se presentaba era determinar los de bienes de consumo y los de capital. Hemos mantenido los automóviles como camionetas y camiones dentro de los bienes de capital que son parte de la formación bruta de capital fijo en transporte. Como en un principio estos rubros no estaban separados (en lo referido a los primeros años de importación de automóviles de combustión interna), puede haber una ligera sobrestimación del sector transporte. 


\section{CUADRO 1. CATEGORIZACIÓN DE LA MAQUINARIA Y EL EQUIPO DE TRANSPORTE}

\begin{tabular}{llcr}
\hline $\begin{array}{l}\text { Tipo de maquinaria } \\
\text { (ejemplos) }\end{array}$ & $\begin{array}{c}\text { Sector } \\
\text { productivo }\end{array}$ & $\begin{array}{c}\text { Categoría } \\
\text { cuentas nacionales }\end{array}$ & $\begin{array}{c}\text { Categoría } \\
\text { cuentas nacionales (II) }\end{array}$ \\
$\begin{array}{l}\text { Bombas para minas } \\
\text { Perforadoras }\end{array}$ & Minería & Maquinaria & $\begin{array}{c}\text { Maquinaria } \\
\text { y equipos }\end{array}$ \\
$\begin{array}{l}\text { Segadoras } \\
\text { Arados }\end{array}$ & Agricultura & Maquinaria & Maquinaria \\
$\begin{array}{l}\text { Máquinas textiles } \\
\text { Máquinas para } \\
\text { producir cerveza }\end{array}$ & Industria & Maquinaria & y equipos \\
Máquinas de escribir & Servicios & Maquinaria \\
& y ciencias & & y equipos \\
Locomotoras & Transporte & Transporte & Maquinaria \\
Carruajes & y equipos & y equipos & Maquinaria \\
\hline
\end{tabular}

Fuentes: elaboración propia con base en Estadística, 1840-1938, y OCDE, Medición, 2009.

artes y oficios vaya desapareciendo. ${ }^{17}$ También es importante recalcar la gran cantidad de elementos que son considerados maquinaria que no cumplen con estas características. Esta condición está determinada porque el rubro "maquinarias, máquinas e instrumentos para las industrias, artes y oficios" permite que ciertos elementos confundan a los trabajadores de aduana. Esta situación se va corrigiendo con el tiempo, pero siempre puede causar problemas en la contabilidad de las series de formación bruta de capital en maquinaria, puesto que algunos anuarios, como por ejemplo los de 1859 y 1883, traen resúmenes de años anteriores, donde obviamente se ha hecho contabilidad en el rubro maquinaria de elementos ajenos a ella.

${ }^{17}$ En el sector maquinarias, en otro tipo de ejemplo, muchas veces se contabilizaban todos los instrumentos musicales (pianos, guitarras, flautas, etc.) y también, los instrumentos científicos. Hacia alrededor de 1900 se comenzó una recolección más "moderna" de los datos, que dejó de lado artículos que estaban completamente alejados de la concepción moderna de maquinaria. No obstante, en esta investigación se han mantenido los instrumentos científicos dentro de la contabilidad. 
Por esta razón es que se ha desistido de ocupar estos resúmenes y sólo se utilizan los anuarios año con año cuando estos están disponibles.

Obtenidas las cifras de maquinaria y equipos por año, en sus respectivas monedas, se procede a su sumatoria, equiparando las cifras a $18 \mathrm{p}$ o 18 pesos oro. Obtenida esta cifra, se utiliza el deflactor de Feinstein ${ }^{18}$ principalmente porque este fue aplicado a las series británicas, que en términos acumulativos es la principal fuente de importación de maquinarias y equipos en Chile.

\section{LAS FUENTES OFICIALES DE LOS PAÍSES MÁS INDUSTRIALIZADOS}

La reconstrucción cuantitativa de la inversión en bienes de equipo de Chile basada en las exportaciones de tales bienes por parte de los países más industrializados ha sido efectuada en diversas partes. ${ }^{19}$ Para el periodo 18901930 los países en cuestión son, en el caso de la maquinaria, Alemania, Estados Unidos y Reino Unido (en adelante G-3) y, en el caso del equipo de transporte, Alemania, Bélgica, Estados Unidos y Reino Unido (G-4). Para el periodo 1870-1889 la estimación se ha realizado sobre la base de las estadísticas del comercio exterior de Estados Unidos y Reino Unido (G-2), mientras que para el periodo 1856-1869 se ha levantado exclusivamente a partir de las estadísticas comerciales británicas (G-1), dadas las deficiencias que presenta la ECE estadunidense hasta 1869. Debido a que el grado de cobertura de las importaciones chilenas de bienes de equipo va decreciendo en cada uno de esos subperiodos, a medida que nos remontamos hacia atrás ha sido necesario reescalar al alza las series elaboradas para los periodos $1856-1869$ y $1870-1889$, lo que no ha presentado ninguna dificultad al tener las magnitudes respectivas de las series en los años de enlace, 1870 y 1890. La reducción de la base estadística -de G-3 o G-4 a G-1- se justifica por la pérdida de relevancia de algunos de los países mencionados como proveedores de bienes de equipo al retroceder en el tiempo, y también a que sus estadísticas resultan poco o nada aprovechables por la falta de datos detallados, por ejemplo, la de Estados Unidos antes de 1870. En cualquier caso, es evidente que el procedimiento de construcción de las series agregadas que hemos seguido presupone que la representatividad y consistencia de los datos del periodo anterior a 1870 y a 1890 son similares a los del periodo posterior. La premisa es, por supuesto, discutible, y seguramente está en el origen de algunas discrepancias detectadas en el ejercicio de contraste que se lleva a cabo en el apartado cuatro. El último tramo

\footnotetext{
${ }^{18}$ Feinstein, National, 1972, y "National", 1988, pp. 257-471.

19 Tafunell, "Capital”, 2009, "Inversión”, 2009, y "Siglo", 2011.
} 
temporal de la estimación (1890-1930) es el más sólido, lo que ha consentido una estimación del valor de las importaciones totales de maquinaria y de otros bienes de equipo aplicando coeficientes de extrapolación de las exportaciones de G-3 y G-4, respecto al total importado, en 1913 y 1925.

A continuación damos cuenta de los principales escollos metodológicos a los que nos hemos enfrentado empleando las fuentes oficiales del comercio exterior de G-3 y G-4. Para evitar reiteraciones, anotamos las características de las fuentes de las ECE que afectan tanto al cálculo de la maquinaria como al del equipo de transporte (el cálculo se ha realizado por separado).

\section{Alemania}

Las ECE alemanas presentan, entre otros, los siguientes problemas: a) no se publicaron estadísticas anuales entre 1914 y $1919 ;{ }^{20}$ b) las estadísticas de 1920, 1921 y 1922 consignan únicamente las cantidades exportadas (quintales métricos o unidades); c) el territorio al cual se refieren las ECE de Alemania no es el mismo antes y después de la primera guerra mundial, pues a raíz de ella, Alemania perdió algunas regiones (Luxemburgo, Alsacia-Lorena, Sarre, Schleswig septentrional, Dánzig, Polonia occidental y Alta Silesia, además de otras poco significativas); $d$ ) las ECE no registran las exportaciones cuyo importe asciende a menos de 5000 marcos; ${ }^{21} e$ ) los valores están denominados en miles de marcos, con lo cual se incurre en errores por imprecisión en ciertos cálculos, como el de pequeños agregados o de valores unitarios de productos concretos, y $f$ ) la estructura de la estadística permanece muy estable a lo largo del periodo, excepto en 1897, y especialmente en marzo de 1906, cuando, con motivo de la nueva ley arancelaria, se multiplica el número de epígrafes, lo que, en la práctica, hace imposible igualar la clasificación anterior con la posterior a esa fecha. ${ }^{22}$

\footnotetext{
${ }^{20}$ Para el periodo bélico solamente se cuenta con cifras sobre el valor y volumen global de importaciones y exportaciones, cuya procedencia son evaluaciones realizadas por autores contemporáneos. Véase Hardach, Primera, 1986, p. 44. Los datos oficiales del comercio de enero a junio de 1914 se publicaron en la estadística mensual, que no hemos podido localizar para estos meses. Por otra parte, la estadística correspondiente a 1921 se refiere al periodo del 1 de mayo al 31 de diciembre.

${ }^{21}$ Las operaciones de menor cuantía se totalizan en un último epígrafe de miscelánea.

${ }^{22}$ Hasta 1905 el número de epígrafes referentes a maquinaria ascendía a 36 . La estructura arancelaria implantada en 1906 -vigente en 1930- amplió los rubros a 145. En lo que se refiere al equipo de transporte, hasta 1896 el número de rubros ascendía tan sólo a nueve. Desde 1897 a 1905 se elevó a 23. La estructura arancelaria implantada en 1906 -vigente en 1930-amplió a 62 los rubros.
} 
Algunos de los inconvenientes que acabamos de indicar son insalvables y deben aceptarse sin más, como por ejemplo, el consecuente al cambio territorial o al vacío informativo del periodo bélico. ${ }^{23}$ Otras limitaciones, que en apariencia suponen un serio impedimento para un cálculo veraz del equipo exportado, como el inciso $d$, tienen en realidad poca importancia: el umbral en cuestión es bajo en relación con el valor unitario de ese tipo de bienes, de manera que en su práctica total cobran existencia identificable en las ECE. En apariencia, la mayor dificultad a la que nos enfrentamos radica en lo señalado en el inciso $f$. No hay modo de evitar la ruptura de las series en 1906. Sin embargo, a la vista de los resultados obtenidos del simple cómputo de los valores registrados en los rubros arancelarios de 1905 y 1906, no hay ningún sesgo significativo. ${ }^{24}$

Por lo que respecta al vacío informativo de 1914 a 1922, hemos optado por lo siguiente: a) extrapolar las magnitudes de 1913 y del periodo mayo-diciembre de 1921 al primer semestre de 1914 y al primer cuatrimestre de 1921, respectivamente $;^{25}$ b) valorar las cantidades exportadas en 1920-1922. No hemos imputado ningún valor, en términos corrientes, al agregado de las cantidades. Lo hemos manejado como un índice de volumen que admite una conversión automática en valores cuando opera con marcos constantes.

\section{Bélgica}

La ECE belga se ha empleado únicamente para estimar la inversión en equipo de transporte en el periodo de 1890 a 1930. Los problemas de

\footnotetext{
${ }^{23}$ Alemania, junto con las otras potencias centrales, fue sometida por los aliados a un bloqueo marítimo que restringió fuertemente su comercio exterior, aunque no logró impedir que mantuviera un cierto nivel de exportaciones a través de puertos neutrales. Al respecto véanse Hardach, Primera, 1986, pp. 23-45, y Stevenson, 1914, 2004, pp. 243-248. El volumen de las exportaciones totales descendió en 1915 hasta 25, respecto a 1913 que eran de 100, y se mantuvo en torno a este nivel hasta 1918, en Wrigley, First, 2000, p. 3. No obstante, todos los indicios que conocemos apuntan a que las ventas alemanas de bienes de equipo a Latinoamérica se redujeron virtualmente a cero en el transcurso del conflicto bélico.

${ }^{24}$ La tasa de variación interanual de 1906 , calculada con la nueva estructura arancelaria guarda relación con la registrada por las exportaciones de bienes de equipo británicas y estadunidenses. Surge entonces la duda de si la clasificación de 1906 no hizo sino aflorar una tipología de nuevos bienes, como el equipo eléctrico, que hasta ese momento estaban comprendidos en categorías que designaban bienes de equipo de manera genérica.

${ }^{25}$ En concreto, hemos supuesto que entre enero y finales de julio de 1914, Alemania exportó la mitad que en el año precedente. Parece un supuesto sensato, a la vista de cómo evolucionaron las ventas a América Latina por parte de las potencias europeas. Tampoco parece descabellado generalizar la actividad exportadora de mayo a diciembre de 1921 a la totalidad de este año; en consecuencia, hemos multiplicado las cifras correspondientes a dichos meses por el coeficiente 1.5 .
} 
esta fuente son, a nuestros efectos, los siguientes: $a$ ) no se publicaron estadísticas entre 1914 y 1919 ; b) desde el 1 de mayo de 1922 la ECE abarca a Bélgica y Luxemburgo, en virtud del tratado de unión comercial firmado por ambos Estados, y c) en noviembre de 1924 se produjo un cambio importante en la estructura de epígrafes de la ECE. Con anterioridad había tenido lugar una paulatina ampliación de los epígrafes, manteniéndose en esencia la clasificación ya vigente en 1890 .

El primer escollo no representa un problema real, puesto que, al igual que Alemania, las exportaciones de bienes de capital belga debieron ser prácticamente nulas durante la guerra y el armisticio. Como en el caso germánico, hemos optado por estimar las exportaciones de enero a julio de 1914 a partir de los valores del año anterior. En cuanto a lo indicado en los incisos $b$ y $c$, representan inconvenientes de menor importancia, ya que, por un lado, la capacidad de fabricación de material de transporte estaba muy concentrada en el territorio belga ${ }^{26} \mathrm{y}$, por otro, las alteraciones en la clasificación de los productos no dan lugar a ninguna inconsistencia en el cálculo del material de transporte exportado.

\section{Estados Unidos}

Las ECE estadunidenses constituyen una fuente de calidad extraordinaria, claramente mayor que las del resto del G-4. Tiene, entre otras virtudes, la particularidad de clasificar los bienes según un criterio coherente y racional desde el punto de vista del análisis económico, y que da lugar a una descomposición de los bienes de equipo que resulta extremadamente útil para el investigador. No obstante, no alcanza a ser una fuente perfecta, puesto que algunas de sus características conllevan problemas metodológicos: a) hasta 1917 no se refiere al año natural sino a uno fiscal que finaliza el 30 de junio (y, lógicamente, que inicia el 1 de julio del año precedente), y, b) no ofrece datos de cantidades para una porción considerable de los rubros arancelarios. La información es heterogénea para el resto (unidades, distintas medidas de peso, capacidad, etc.). Por consiguiente, sólo pueden obtenerse magnitudes agregadas en valor (expresadas en dólares).

La segunda limitación implica que la cuantificación de los bienes de equipo exportados por G-3 solamente puede hacerse en valores monetarios. Los índices cuánticos o cualquier tipo de series de volumen quedan

\footnotetext{
${ }^{26}$ De hecho, las ECE de los años inmediatamente posteriores a la unión comercial proporciona datos desagregados para los dos países, aunque compilarlos exige una tarea más ardua y tediosa que la acometida en esta investigación. Esta información revela la extremadamente desigual importancia de los dos socios comerciales en el terreno de la fabricación de equipo de transporte.
} 
descartados justamente porque se carece de la información necesaria en el caso de las exportaciones estadunidenses. La primera limitación implica que, dada la inexistencia de estadísticas mensuales, el ajuste de las cifras de exportación de años fiscales a naturales debe realizarse promediando pares consecutivos de años fiscales, asignando el valor resultante al primero de ellos. La información disponible no admite otra solución, pero no por ello debe pasarse por alto que se trata de una estimación sujeta a márgenes de error. El método de cálculo presupone que en cada año la actividad exportadora se desenvolvió por igual en ambos semestres, lo que sólo sería cierto si no hubiese habido estacionalidad ni ciclos económicos. Como en realidad no es así, no cabe esperar que las magnitudes calculadas a partir de la estadística estadunidense para el periodo anterior a 1918, una vez ajustadas a los años de calendario, expresen con total exactitud el montante anual de su comercio exterior.

\section{Reino Unido}

La estadística británica plantea muchos más problemas que las ECE de Alemania y Estados Unidos. Los principales inconvenientes son los siguientes: a) la descomposición de la maquinaria exportada es mínima, incluso para los países, como Chile, para los cuales la ECE ofrece más información. La falta de desglose estadístico no permite tener la seguridad de que a lo largo del periodo estudiado los datos abarquen el mismo tipo de bienes; b) la clasificación de los bienes de capital entre las distintas categorías desplegadas en la estadística y el desglose de las mismas no permanece inalterable a lo largo del periodo. Los cambios más importantes tuvieron lugar en 1909 y 1920 (antes de este último año las locomotoras de vapor estaban incluidas en el total de maquinaria). Pero debe aclararse que, en realidad, dejando de lado la clasificación de la locomotoras, los cambios en la estructura de la estadística no parecen tener un efecto distorsionador sobre nuestro cálculo; ${ }^{27}$ c) la ECE da noticia del valor de los bienes exportados (en libras esterlinas), pero en muchas ocasiones no informa de las cantidades exportadas (equipo eléctrico, herramientas); d) para el periodo anterior a 1890 los datos sobre herramientas y utensilios, material eléctrico distinto a la

\footnotetext{
${ }^{27}$ Por ejemplo, el gran cambio en la clasificatoria introducido en 1920 no conlleva ninguna ruptura en lo referente a los medios de transporte: la suma de las tres categorías que hasta este año cobijaban los diversos medios de transporte (carriages, locomotives y ships and boats) es casi equivalente a la categoría omnicomprensiva (vehicles) instaurada en dicha fecha. Ciertamente la omisión del grupo de embarcaciones (no militares) en las ECE anteriores a 1899 ocasiona una ruptura en este año. Hemos tomado la opción de extrapolar la proporción entre el valor de las exportaciones de buques y de los otros medios de transporte en 1899-1909 al periodo 1890-1898.
} 
maquinaria eléctrica y equipo de comunicaciones son tan escasos, o puramente inexistentes, que no permiten calcular un agregado de maquinaria que incluya este tipo de bienes de equipo, y e) en el periodo anterior a 1890 la fuente no siempre proporciona datos sobre las locomotoras exportadas. Hemos tenido que cubrir las lagunas con estimaciones bastante arriesgadas -especialmente en el periodo anterior a 1862, respecto al cual carecemos de información sobre los medios de transporte exportados-, lo que afecta a los agregados calculados para la maquinaria y el equipo de transporte, aunque no para los bienes de equipo en su conjunto, que, lógicamente, agrupan a ambas categorías.

¿Qué datos de las estadísticas de G-3 y G-4 han sido seleccionados, para después ser agregados? Responder a esta pregunta viene a ser lo mismo que definir los conceptos operativos de "maquinaria y otros equipos" y "medios de transporte" que hemos manejado. En el caso de la maquinaria y equipos afines, hemos calculado el gasto realizado en la adquisición de maquinaria y herramientas, así como sus partes, piezas y accesorios, ya sean de aplicación general, o bien, para usos específicos (agrícola, minera, manufacturera, equipo de oficina); maquinaria y equipo eléctricos; equipos y aparatos de comunicaciones; motores y bombas, así como otros aparatos empleados como medios de producción de toda clase de bienes. ${ }^{28}$ En el caso del equipo de transporte, la evaluación comprende toda clase de equipo de transporte móvil, esto es, el material rodante ferroviario y tranviario, las embarcaciones de cualquier género excepto los buques de guerra, los carros, carruajes, bicicletas y los vehículos propulsados por un motor de combustión interna, excluyendo los automóviles de turismo, es decir, los vehículos comerciales (furgonetas, camiones, omnibuses, etc.). No han sido objeto de evaluación los medios de transporte tradicionales, tales como los carros y los animales de tiro.

Es importante aclarar que no siempre hemos contado con datos fehacientes sobre las exportaciones de bienes de equipo de G-3 y G-4. Las lagunas estadísticas, en realidad, se concentran en los primeros años en el capítulo de los medios de transporte, cuando solamente nos apoyamos en la estadística británica. Hasta entrada la década de 1880 la información que esta proporciona sobre las exportaciones de equipo ferroviario es discontinua o fragmentaria, y simplemente inexistente para antes de 1861, lo que nos ha obligado a estimar el valor de esas exportaciones a partir de la

${ }^{28}$ Siguiendo el criterio de cuentas nacionales de Naciones Unidas, la maquinaria y el equipo comprende el gasto en mobiliario, instrumentos musicales, ópticos y de precisión, relojes y el instrumental médico, lo cual plantea dificultades tanto para captar todos esos bienes en las ECE como, sobre todo, para discernir aquellos que se destinaron a un uso productivo de aquellos otros destinados al consumo de los hogares. Por ello, no hemos tomado en cuenta esos bienes en la cuantificación. 
evolución de la red ferroviaria. Los valores estimados de la inversión en equipo de transporte para el periodo previo a 1870 son menos fiables que los posteriores a esta fecha, pues a partir de ella contamos con la estadística estadunidense, cuya información aparentemente no presenta ninguna laguna ni inconsistencia en la categorización de los bienes.

Una vez que hemos obtenido las magnitudes de la maquinaria, por un lado, y el equipo de transporte, por otro, exportados por Alemania, Estados Unidos, Reino Unido y, eventualmente, Bélgica, los pasos que hemos dado para completar la estimación han sido muy sencillos: la pura agregación de las series de cada tipo de bienes de equipo, previa conversión de tales magnitudes a una moneda común, la libra esterlina, utilizando los tipos de cambio. ${ }^{29} \mathrm{El}$ agregado resultante se ha deflactado utilizando el índice de precios de los bienes de equipo británicos para expresar el valor de las exportaciones en términos constantes. ${ }^{30}$

\section{COMPARACIÓN DE RESULTADOS}

Los apartados anteriores tienen el carácter de nota metodológica sobre las estadísticas oficiales del comercio exterior como fuente para cuantificar el gasto en bienes de equipo de una economía dependiente casi enteramente de las importaciones de ese tipo de bienes, como fue la chilena en la época anterior a la gran depresión de los años treinta. ${ }^{31}$ De la descripción y crítica

${ }^{29}$ Las series de tipos de cambio en Carter, Historical, 2006, pp. 565-567, siguiendo las recomendaciones de su compilador, Lawrence $\mathrm{H}$. Officer, cuando existían diversas posibilidades. La valoración de las exportaciones germánicas habría podido resultar complicada en el paréntesis hiperinflacionario de 1920-1923, pero la ausencia de una valoración nominal en las ECE alemanas ha obviado el problema al darnos la posibilidad de construir un índice en cadena basado en las cantidades.

${ }^{30}$ El índice empleado para la maquinaria en el periodo de 1856 a 1920, en Feinstein, "National", 1988, pp. 470-471, ha sido empalmado en 1920 con el índice que elaboró anteriormente este autor en National, 1972, pp. 136-137, tras haber convertido ambos a base 100 en 1913. En el caso de los medios de transporte, para el periodo de 1856 a 1920 hemos utilizado el índice de precios sintético de material rodante ferroviario, buques y vehículos construido por Feinstein, "National", 1988, pp. 470-471. Hemos empalmado la serie anterior, con base en 1913, con otra que hemos elaborado para 1920-1930 basándonos en los índices de precios de material rodante ferroviario, buques y vehículos calculados por Feinstein National, 1972, pp. 136-137, ponderándolos según el peso relativo que tuvieron en 1925 esos bienes en las exportaciones británicas a América Latina de material de transporte.

${ }^{31}$ Los datos de la década de 1920 muestran que en sus mejores años la participación de la maquinaria nacional en el total de la formación bruta de capital en maquinaria no alcanza 3\%. Como se ha explicitado someramente, en ningún momento de su historia, Chile ha contado con una participación destacada en la elaboración de su maquinaria. Otros autores, especialmente Ortega y Salazar, han tratado de cambiar esta percepción por medio del argumento de la producción de locomotoras, las maestranzas y el trabajo en vagones, pero no han elaborado las series de producción para demostrarlo. El primer autor valoriza de forma cualitativa la producción de 
de las fuentes, así como de las opciones que hemos tomado al manejar los datos para calcular la magnitud anual de la inversión se desprende que las cifras finalmente establecidas usando las estadísticas comerciales de G-3 y G-4 y la estadística de Chile es improbable que sean absolutamente coincidentes. La cuestión que debemos dilucidar ahora, la cual es el verdadero núcleo de este artículo, es: ¿cuán importantes son las discrepancias entre ambas aproximaciones cuantitativas? Esta pregunta básica dará lugar a su vez a que nos formulemos diversos interrogantes: ¿̇las diferencias han tenido la misma significación a lo largo del periodo objeto de análisis?; de no ser así, ¿cuáles son las causas explicativas del contraste entre unas y otras etapas? Y en cuanto a las disparidades que eventualmente tengan un carácter "permanente", ¿̇ué factor o factores las han originado, hipotéticamente? Ahora observemos sin más preámbulos los resultados.

Las series que confrontamos en la gráfica 1 , y recogemos en el cuadro 2 , como puede verse, están expresadas en números índice, con base en 1913, de la inversión en términos constantes (en valores de este mismo año). Omitimos las series en valores corrientes porque no aportan nada a nuestro ejercicio comparativo al haber sido deflactadas ambas elaboraciones por los mismos índices de precios. El lector puede extrañar un contraste directo entre las magnitudes absolutas de nuestras estimaciones -previa conversión, lógicamente, a una unidad monetaria común. Lo hemos descartado porque complicaría la comparación, al incorporar con toda seguridad un factor de diferenciación: el distinto sistema de valoración de los bienes comerciados, con base en el free on board, ${ }^{32}$ en las estadísticas de los exportadores, y en el cost insurance freight ${ }^{33}$ en la del importador. Por consiguiente, nuestro ejercicio comparativo se ciñe a los números índice, que son, al fin y al cabo, trasunto de las tasas de crecimiento, lo cual, a la postre, significa que tomamos en cuenta las diferencias de nivel entre ambas series que pudieran derivarse de una discrepancia en su ritmo de crecimiento que fuera temporalmente sostenida. ${ }^{34}$

Una primera lectura de la gráfica 1 , que preste atención únicamente a los grandes movimientos, podría resumirse en una notable divergencia entre 1862 y 1877 y un fuerte paralelismo a partir de entonces hasta el

\footnotetext{
bienes de capital de Chile, pero a la vez sentencia "con los datos actuales, es imposible estimarla monetariamente". Ducoing, "Inversión”, 2011. Véanse también Ortega, Chile, 2005, y Salazar, Mercaderes, 2009.

${ }^{32}$ FOB, "franco a bordo".

${ }^{33}$ CIF, "Coste, seguro y flete".

${ }^{34} \mathrm{Si}$ las tasas de crecimiento fueran temporalmente sostenidas, la conclusión del trabajo sería que no podrían ser utilizadas ambas series indistintamente para medir el esfuerzo inversor de la economía chilena en el periodo comprendido entre 1856-1930.
} 


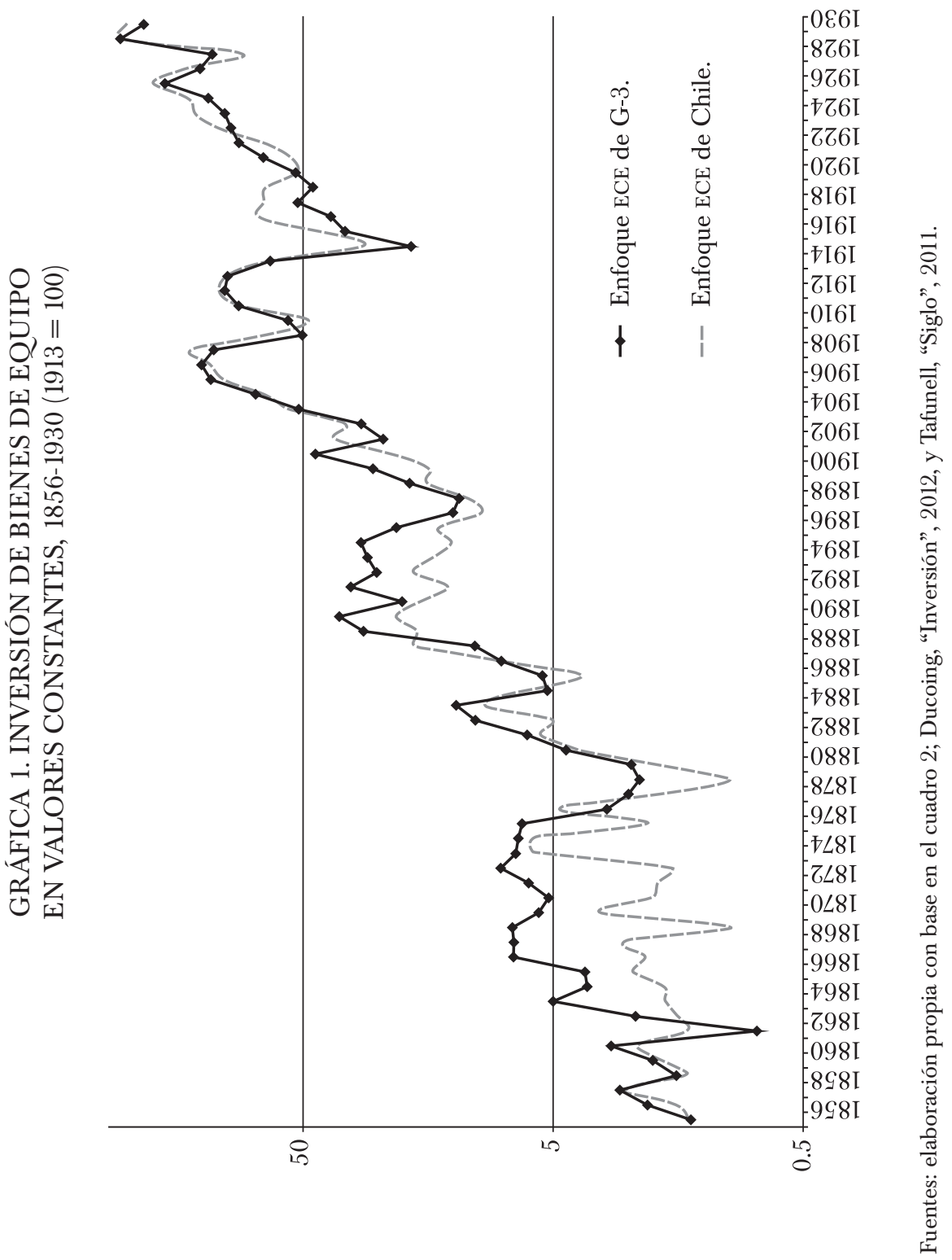


CUADRO 2. TASAS DE CRECIMIENTO ANUAL DE LA INVERSIÓN EN BIENES DE EQUIPO (PORCENTAJES) ${ }^{a}$

\begin{tabular}{lcc}
\hline Periodo & Enfoque ECE de Chile & Enfoque ECE de $G-3$ \\
$1856-1930$ & 7.5 & 6.4 \\
$1856-1877$ & 3.9 & 7.7 \\
$1877-1930$ & 8.1 & 7.1 \\
$1877-1890$ & 19.2 & 19.6 \\
$1890-1913$ & 11.5 & 8.0 \\
$1913-1929$ & 8.4 & 10.4 \\
\hline
\end{tabular}

${ }^{a}$ Calculadas mediante ajuste lineal de los logaritmos de los valores.

Fuente: elaboración propia con base en los datos del apéndice.

final del periodo examinado. El cuadro 2 confirma y precisa esta primera apreciación visual del perfil de las curvas.

El crecimiento secular del conjunto del periodo es del mismo orden en las dos estimaciones; estas están distanciadas por un punto porcentual de crecimiento anual, tasa que se reduciría a tan sólo $0.3 \%$ si consideráramos el bienio inicial y final de ese periodo secular. ${ }^{35}$ Se observa prácticamente la misma similitud al considerar el largo medio siglo comprendido entre 1877 y 1930. Por el contrario, la discordancia se manifiesta en el periodo de 1856 a 1877. Si descomponemos el periodo posterior en tres subperiodos, atendiendo a las grandes fases de crecimiento, podemos delimitar una cronología cuyos picos serían 1877, 1890, 1913 y 1929. Pues bien, el cuadro 2 revela una coincidencia bastante aceptable en las tendencias subyacentes de ambas series. De 1877 a 1890 son totalmente parejas, mientras que en los dos subperiodos siguientes se registra una desproporción moderada en el ritmo de crecimiento de fondo, alternándose las posiciones de mayor y menor dinamismo de las series. Parece bastante claro, al observar la gráfica, que el origen de tal divergencia radica en las fluctuaciones de más corto plazo, las cuales no tienen ni la misma amplitud e intensidad ni tampoco cronología idéntica en las dos estimaciones. Un indicio en este sentido se encuentra en el hecho de que la pendiente de las tendencias de los subperiodos indicados es muy semejante si se calcula tomando en consideración

\footnotetext{
${ }^{35}$ Como se aprecia claramente en la gráfica 1, los niveles relativos de ambas series entre 1856 y 1857,1929 y 1930 son virtualmente idénticos. La divergencia, moderada, registrada en el cuadro 2 obedece al impacto en la tendencia a largo plazo de los índices de su trayectoria disímil en los años 1860 y primera mitad de los 1870 .
} 
solamente los puntos extremos señalados. ${ }^{36}$ Por lo tanto, desde la perspectiva de los movimientos a largo plazo, es preciso distinguir la evolución anterior a 1877 de la posterior. Hasta esta fecha, nuestras reconstrucciones estadísticas exhiben mayores diferencias que similitudes. Naturalmente, no basta con reconocerlo; hay que averiguar cuál es la causa, o por lo menos conjeturar cuáles son los potenciales elementos explicativos del desacuerdo. Lo haremos en seguida. En todo caso, antes de pasar a ello conviene dejar sentado que a partir de 1877 los dos enfoques de cuantificación de la inversión tienen una gran afinidad. Lo cual, por supuesto, resulta reconfortante, pues avala la consistencia de nuestras dos elaboraciones, que han sido realizadas de forma totalmente independiente. La gráfica 2 aporta una evidencia suplementaria y, a nuestro juicio, contundente en este sentido.

En efecto, la gráfica 2, en la que observamos de manera directa el grado de asociación estadística entre ambas series, muestra que esta es ciertamente elevada. Sin hacer distingos entre periodos, tomando en su totalidad las series, descubrimos que los pares de valores de cada año tienden a situarse cerca de la recta bisectriz. Con esta evidencia empírica no pretendemos encubrir las discrepancias, sino simplemente contextualizarlas para calibrar su significación global. La gráfica 2 sugiere que esta es de limitado alcance, que la nota dominante en el perfil de las series es su semejanza. Pero no vamos a recrearnos en este aspecto positivo, que, lógicamente, confiere fiabilidad a las estimaciones realizadas; antes al contrario, dando un sesgo crítico al ejercicio desarrollado en este artículo, en lugar de insistir en los paralelismos nos centraremos en examinar acuciosamente las discordancias entre las estimaciones, siempre que sean estadísticamente relevantes.

La gráfica 3 brinda una imagen cruda de las discordancias entre las series. Lo primero que debemos advertir es que estas se ven magnificadas y multiplicadas por la asincronía en las fluctuaciones de corto plazo de los dos agregados: un simple adelanto de un año de uno de ellos tiende a traducirse en una fuerte variación en la ratio, puesto que la inversión muestra ser muy volátil. Es más, el violento repunte en la ratio suele ir precedido o seguido de una no menos intensa caída, en lo que no es otra cosa que un automático efecto eco de la mencionada asincronía cíclica. Ejemplos de este fenómeno se descubren en 1884-1885 -o bien, 1885-1886-, en 18881889, 1891-1892, 1907-1908, etc. Desde una perspectiva de mayor alcance, es significativo que a partir de 1874, y con la excepción del ciclo de la primera guerra mundial, el promedio de la ratio en periodos largos (de un

\footnotetext{
${ }^{36}$ Medida de este modo, la tasa de crecimiento entre 1890 y 1913 es de 4.6\% y 3.7\%, según la elaboración basada en las ECE G-3 y ECE Chile, respectivamente, mientras que para 1913-1929 las dos elaboraciones arrojan el mismo resultado: un incremento anual de 6.4 por ciento.
} 


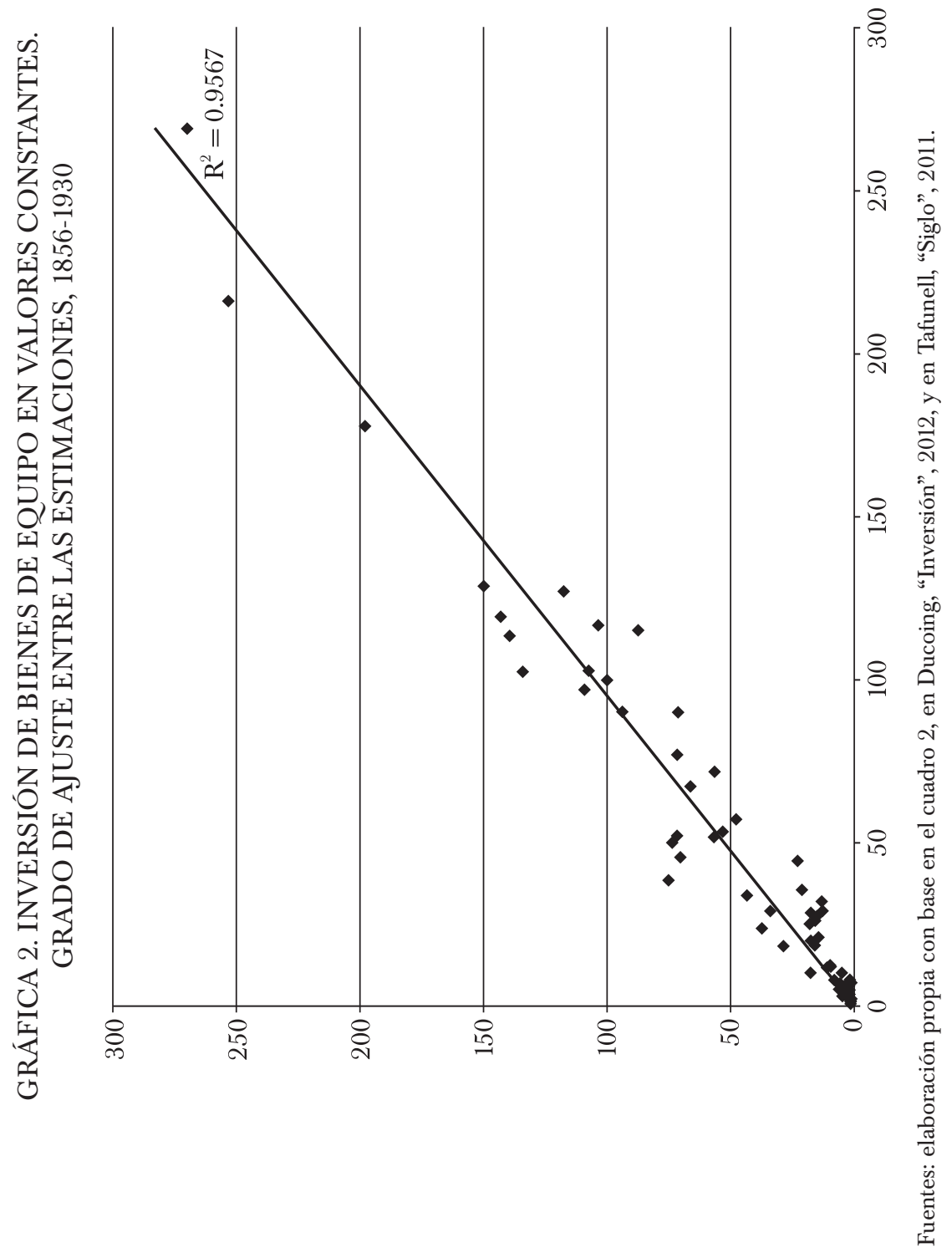




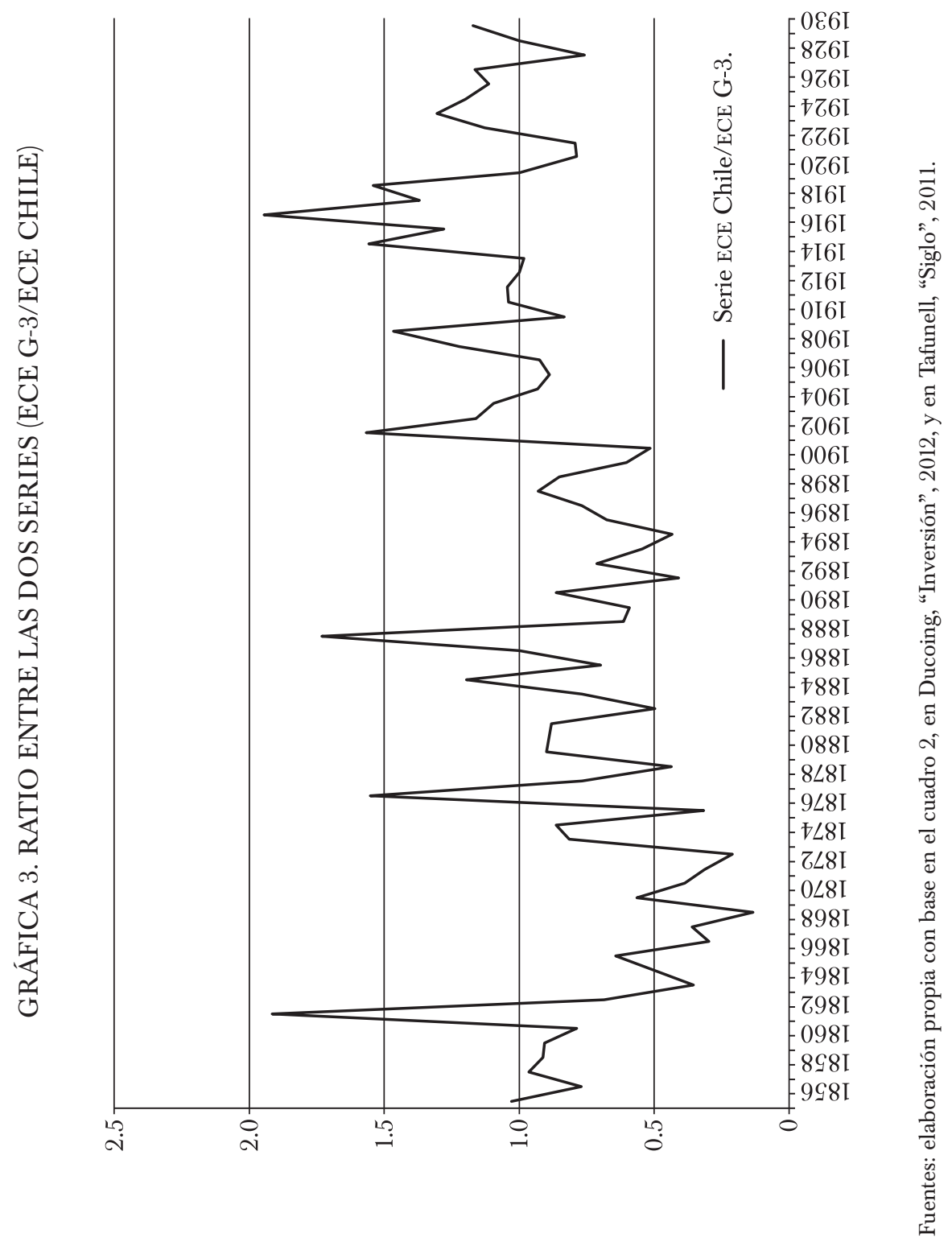


decenio o más) tienda a un valor próximo a la unidad, es decir, tienda a compensarse.$^{37}$ Esta constatación aconseja aislar el efecto de desfasamiento de las series mediante un filtro estadístico.

A través de una media móvil trienal centrada, reafirmamos plenamente que las grandes diferencias observadas en las series están concentradas en el periodo comprendido de 1863 a 1875, y, también, en menor grado, en el periodo de 1915 a 1919. En los años restantes, pese a existir algunas diferencias puntuales, es patente el paralelismo entre ambas series (véase gráfica 4$)$.

\section{HIPÓTESIS EXPLICATIVAS DE LAS DIFERENCIAS}

El análisis descriptivo del apartado anterior ha ofrecido una visión general de las concordancias y discordancias entre las dos estimaciones. A continuación, nos detendremos a formular hipótesis explicativas de las diferencias fundamentales entre ambas series en los dos periodos en los que se concentran las disparidades más acusadas, entre 1862-1876 y 1914-1918.

En 1862-1876 se observan diferencias notorias y falta de concordancia en general. No sólo divergen ambas series en tendencias; los niveles varían considerablemente, a lo largo de un espectro de ratios tan amplio que alcanza un valor máximo de 1.92 (1862) y un mínimo de 0.13 (1869). Sin poder brindar una interpretación definitiva de cuáles son las causas de tamañas diferencias, se puede aventurar una serie de hipótesis para explicarlas. En primer lugar, la ECE de Chile recoge la totalidad de la maquinaria internada en Chile, mientras que las ECE de G-3, lógicamente, sólo dan cuenta de las exportaciones de esos tres países a Chile. Además, se da la circunstancia que, como hemos señalado antes, hasta 1869 nuestra estimación basada en las fuentes extranjeras se apoya exclusivamente en las exportaciones británicas. Por ello, resulta necesario y apremiante responder a la interrogante: ¿cuán representativos eran los bienes de equipo británicos, en particular, y los de G-3, en general, en las compras al exterior de ese tipo de bienes realizados por Chile? La respuesta se encuentra en el cuadro 3.

Como muestra este cuadro, la cuota de mercado de G-3 era ya muy elevada en $1855(86.7 \%)$, y subiría todavía más en diversos momentos, sobrepasando con creces el nivel de $90 \%$. Desde luego, esto significa que G-3 tenía un peso muy predominante, abrumador, en la provisión de maquinaria y equipo para la economía chilena, al igual que para el resto de

${ }^{37}$ Los valores medios y, entre paréntesis, la desviación estándar, son 1874-1897, 0.79 (0.33); 1898-1914, 1.00 (0.25), y 1920-1930, $1.04(0.18)$. 


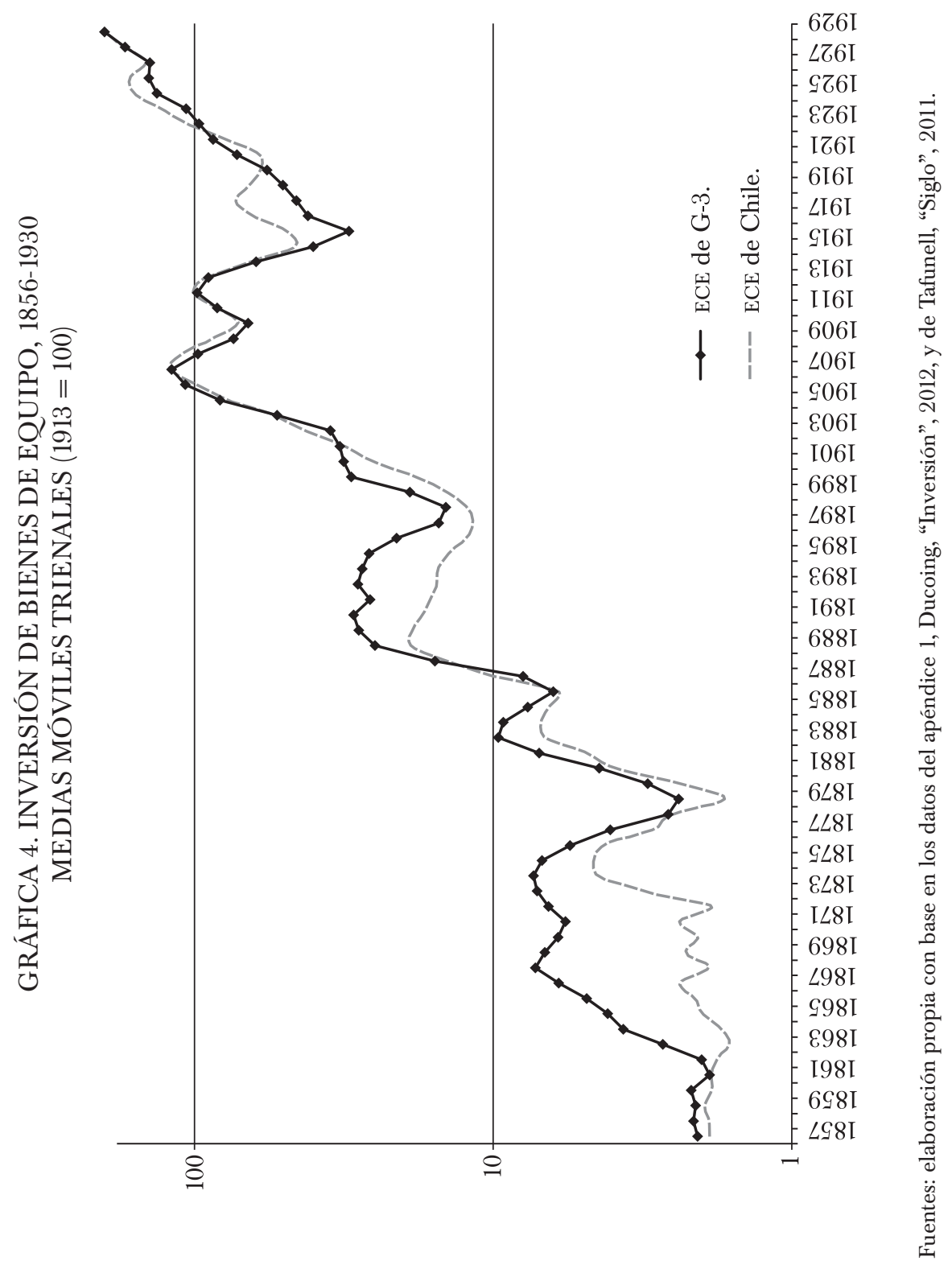




\section{CUADRO 3. PARTICIPACIÓN EN LAS IMPORTACIONES CHILENAS \\ DE MAQUINARIA Y EQUIPOS \\ (PORCENTAJES)}

\begin{tabular}{lcrcccc}
\hline & & & & Reino & Estados & \\
Años & Francia & Otros & Alemania & Unido & Unidos & G-3 \\
1855 & 10.93 & 2.28 & 5.46 & 66.04 & 15.29 & 86.74 \\
$1871^{\text {a }}$ & s. d. & 22.66 & s. d. & 36.97 & 40.37 & 77.34 \\
1892 & 2.63 & 5.12 & 15.30 & 51.61 & 25.37 & 92.28 \\
1909 & 3.52 & 14.03 & 18.35 & 32.53 & 31.62 & 82.47 \\
1915 & 1.36 & 7.60 & 17.33 & 18.43 & 55.28 & 91.04 \\
1928 & 1.88 & 8.24 & 24.59 & 16.99 & 48.31 & 89.98 \\
\hline
\end{tabular}

a Sólo información de Estados Unidos y Reino Unido.

Fuentes: para 1855, Anuario de Comercio Exterior, para 1871 y 1892, Foreign, 1870-1930, en específico los años 1909 y 1914, y para 1928, Anuario, 1910-1930.

las economías latinoamericanas. ${ }^{38}$ En 1855 , los productos británicos también tenían una participación mayoritaria en las importaciones chilenas $(66 \%)$, aunque fueron perdiendo mucha cuota de mercado con el paso del tiempo. Pero ahora nos interesa llamar la atención sobre otro aspecto del cuadro 3: la participación de G-3, y probablemente G-1, fue fluctuante. No es descartable que la evolución de las importaciones totales de maquinaria difiera de la evolución marcada por las exportaciones de G-3 (más aún de G-1) en el caso de que la cuota de mercado de estas se alterase significativamente, de forma coyuntural, debido a una compensación entre el peso relativo de las exportaciones de G-3 (G-1) y del resto del mundo. Esto es algo que seguramente pudo ocurrir con mayor probabilidad en las tres primeras décadas del periodo examinado que más adelante, cuando Francia y otras naciones europeas perdieron relevancia como proveedores de maquinaria, mientras Alemania queda incorporada efectivamente a nuestra base de datos de G-3.

Una segunda hipótesis explicativa de las diferencias observadas entre 1862 y 1877 radica en las lagunas estadísticas de la fuente británica en lo que respecta al equipo de transporte. Ante la ausencia -total o parcialde datos en las ECE británicas sobre las exportaciones a Chile de material rodante ferroviario para algunos de esos años, no nos ha quedado otro

${ }^{38}$ Tafunell, “Capital”, 2009, e "Inversión”, 2009. 
remedio que cubrir los vacíos informativos con estimaciones que, sin duda, adolecen de importantes márgenes de error. ${ }^{39}$

Una tercera hipótesis explicativa de las discrepancias reside en el hecho de que la estimación basada en G-3 no incluye las herramientas para el periodo anterior a 1870, mientras que la ECE de Chile comprende las herramientas para artesanos y agricultores. ${ }^{40}$ Por lo tanto, el nivel inicial de la elaboración basada en ECE de Chile debería ser más alto y las fluctuaciones de dicha elaboración podrían ser disimiles con la elaboración derivada de las ECE de G-3. Una última explicación plausible para el periodo más conflictivo de la contrastación, estriba en la diferencia de avalúos en los puertos chilenos respecto a las facturas de exportación británicas y estadunidenses. Hasta 1883, las maquinarias no pagaron derechos de importación en Chile; en una medida proteccionista con la intención de favorecer a la industria metalmecánica, alentada por la Sociedad de Fomento Fabril,$^{41}$ se impuso un aumento del impuesto específico a las maquinarias, correspondiente a 15\% del valor CIF, por lo tanto, la introducción de los bienes de capital comenzó a ser consignada contra factura, disminuyendo la arbitrariedad en la estimación del valor por medio de avalúos. No es casualidad que las mayores diferencias en el ratio de las series se presenten en el periodo de 1856 a 1883.

Un último elemento que afecta a la fiabilidad de la contrastación entre ambas series deriva del nivel de detalle de la estadística británica. $\mathrm{Al}$ principio, en 1856, la ECE no consignaba más que machinery and mill work, obviando algunos rubros que sí son consignados en la estadística chilena, como los carruajes y carretillas en la sección transporte y las herramientas de artesanos, jardineros y mineros. Hacia $1865^{42}$ las estadísticas británicas poseen una mayor desagregación que permite la inclusión de elementos contabilizados en la estadística chilena.

Examinemos a continuación lo que subyace tras los datos de 1914 a 1918, el segundo episodio de fuerte divergencia entre nuestras estimaciones. Respecto a esta coyuntura, no cabe duda que obedece a una causa singular: la perturbación en el comercio internacional provocada por la

${ }^{39}$ Las lagunas estadísticas que han debido salvarse al hacer el cálculo del valor global de los bienes de equipo son las referentes a vagones de mercancías y pasajeros. Las lagunas existentes acerca de las locomotoras son sólo aparentes al estar estas incluidas en los totales de maquinaria. Para mayores detalles, véase Tafunell, "Inversión", 2009.

${ }^{40}$ Entre 1870 y 1889 la cobertura es sólo parcial, ya que sólo disponemos de datos sobre las herramientas exportadas por Estados Unidos de Norteamérica.

${ }^{41}$ Fundada en 1883, año de la finalización de la guerra del Pacífico.

${ }^{42} \mathrm{El}$ Annual Statement of the Trade de 1865 incluye en sus nuevas categorías: agricultural implements, coaches, chariots, chaises, and railway carriages for passengers, railroad wheels and axles, steam engines, not locomotive, steam engines or parts of steam engines of locomotive. Se mantienen en el resumen final por países las descripciones machinery not being steam engines y steam engines. 
guerra mundial. Cualquier elaboración que se base en los datos estadísticos de los proveedores tradicionales, posiblemente incurra en una infravaloración de la compra de equipo durante ese ciclo bélico. Chile, como las demás economías latinoamericanas, se vio impelido a buscar fuentes de aprovisionamiento alternativas para sortear la interrupción del comercio entre ambos lados del Atlántico. Ahí es preciso puntualizar que el cuadro 3 da una imagen que difícilmente se ajusta a la realidad, pues otorga a Alemania una participación probablemente superior a la real, aun tomando en cuenta el comercio de tránsito a través de países neutrales. Sea como fuere, no deberíamos atribuir el máximo valor explicativo a la conjetura de la diversificación comercial. Esto es lo que se desprende de un rápido ejercicio de contraste estadístico que hemos efectuado sobre Argentina y Brasil. ${ }^{43}$ Entonces, ¿cuál es el factor perturbador que ocasiona la diferenciación de ambas estimaciones? Con toda probabilidad, reside en la desigual evolución del valor de los bienes en los países de origen y en el de destino. Y esto puede deberse tanto al valor atribuido por las autoridades a unos bienes cuyo precio sufrió fuertes variaciones, en un contexto altamente inflacionista, como a los tipos de cambio aplicados, y, sobre todo, a la incidencia de los costos de transporte, que ascendieron vertiginosamente por las dificultades y riesgos de la navegación marítima durante el conflicto (recuérdese que G-3 valora los bienes en términos FOB, mientras que Chile lo hace en términos CIF).

\section{CONCLusiones}

Del análisis comparativo de las series elaboradas a partir de las ECE de Chile y del G-3 se presentan las siguientes conclusiones. En primer lugar, en el largo plazo ambas series evolucionan con una tendencia concordante. Esto significa que los investigadores que basen sus trabajos en comercio internacional pueden utilizar indistintamente ambas fuentes. Esto es tanto más cierto cuanto mayor sea el nivel de agregación estadística. Aunque los bienes que nosotros hemos abarcado comprenden un gran número de rubros arancelarios, cabe descartar que en las ECE de alguno de los países,

\footnotetext{
${ }^{43}$ En el caso de Brasil, las compras de bienes de equipo calculadas a partir de los datos de la estadística propia, disminuyeron prácticamente en la misma proporción que la resultante de hacer el cómputo con las estadísticas de G-3. En concreto, los valores respectivos son los siguientes (sobre $1913=100): 1914,36.2$ y $44.7 ; 1915,12.3$ y $12.7 ; 1916,15.7$ y $16.3 ; 1917,19.9$ y 17.7 , y $1918,18.5$ y 14.5. En el caso de Argentina, el uso de las ECE de G-3 conduce a una mayor sobrevaloración de la caída, aunque la distancia que separa ambos índices no es muy grande: 1914, 58.3 y $59.6 ; 1915$, 29.5 y $24.4 ; 1916,31.4$ y $19.6 ; 1917,28.7$ y 13.7 , y $1918,22.6$ y 15.7.
} 
alguno de esos bienes haya escapado a nuestros cálculos por figurar en renglones de bienes no identificables o inclasificados. ${ }^{44}$

En segundo lugar, no podemos obviar que en el periodo comprendido entre 1862 y 1877 las diferencias son muy acusadas. No es el objetivo de este artículo demostrar cuál es la causa precisa de esas diferencias, pero hemos formulado varias hipótesis explicativas que tendrán que ser analizadas a profundidad más adelante. La hipótesis más certera que podemos manejar es que los rubros considerados como maquinaria y equipos por ambas fuentes estadísticas difieren de forma considerable en este periodo, haciendo incorrecto el análisis comparativo a menos que se hiciera una contabilidad desagregada completa, situación que es imposible por medio de las fuentes británicas y estadunidenses por las razones enunciadas en el apartado anterior.

Por último, es importante recalcar que más que trabajar en exclusiva con una u otra fuente estadística, el investigador del comercio internacional latinoamericano del periodo anterior a 1930 debería manejar al mismo tiempo las estadísticas de los principales países proveedores y de las fuentes locales, no tanto por los problemas de calidad que las caracterizan, sino por su complementariedad. Esto es particularmente importante al momento de estudiar categorías específicas de la maquinaria, como puede ser su fuerza motriz o el sector productivo al cual están destinadas.

\section{APÉNDICE}

IMPORTACIONES DE BIENES DE EQUIPO EN CHILE EN TÉRMINOS REALES $(1913=100)$

\begin{tabular}{lccccc}
\hline Año & ECE Chile & ECE socios & Año & ECE Chile & ECE socios \\
1856 & & & & & \\
1857 & 1.4 & 1.4 & 1894 & 15.1 & 27.6 \\
1858 & 1.6 & 2.1 & 1895 & 12.7 & 29.3 \\
1859 & 2.6 & 2.7 & 1896 & 14.3 & 21.2 \\
1860 & 1.5 & 1.6 & 1897 & 9.7 & 12.6 \\
1861 & 1.8 & 2.0 & 1898 & 11.0 & 11.9 \\
1862 & 2.3 & 2.9 & 1899 & 15.9 & 18.7 \\
1863 & 1.5 & 0.8 & 1900 & 15.7 & 26.3 \\
1864 & 1.6 & 2.3 & 1901 & 22.8 & 44.6 \\
& 1.8 & 5.0 & 1902 & 37.4 & 23.8
\end{tabular}

${ }^{44}$ Esto es particularmente cierto para los años iniciales cubiertos en esta comparación. 


\begin{tabular}{|c|c|c|c|c|c|}
\hline Año & ECE Chile & ECE socios & $A \tilde{n} o$ & ECE Chile & ECE socios \\
\hline 1865 & 1.8 & 3.6 & 1903 & 33.8 & 29.2 \\
\hline 1866 & 2.4 & 3.7 & 1904 & 56.7 & 51.9 \\
\hline 1867 & 2.1 & 7.2 & 1905 & 71.6 & 77.1 \\
\hline 1868 & 2.6 & 7.2 & 1906 & 103.6 & 116.8 \\
\hline 1869 & 1.0 & 7.3 & 1907 & 117.6 & 127.2 \\
\hline 1870 & 3.2 & 5.7 & 1908 & 139.3 & 113.6 \\
\hline 1871 & 2.0 & 5.2 & 1909 & 73.5 & 50.2 \\
\hline 1872 & 1.9 & 6.2 & 1910 & 47.7 & 57.3 \\
\hline 1873 & 1.7 & 8.1 & 1911 & 93.7 & 90.3 \\
\hline 1874 & 5.7 & 7.0 & 1912 & 107.4 & 102.9 \\
\hline 1875 & 5.9 & 6.9 & 1913 & 100.0 & 100.0 \\
\hline 1876 & 2.1 & 6.6 & 1914 & 66.3 & 67.4 \\
\hline 1877 & 4.7 & 3.0 & 1915 & 28.6 & 18.4 \\
\hline 1878 & 1.9 & 2.5 & 1916 & 43.3 & 34.0 \\
\hline 1879 & 1.0 & 2.2 & 1917 & 75.1 & 38.6 \\
\hline 1880 & 2.2 & 2.4 & 1918 & 71.6 & 52.3 \\
\hline 1881 & 3.9 & 4.4 & 1919 & 70.3 & 45.6 \\
\hline 1882 & 5.6 & 6.3 & 1920 & 53.1 & 53.5 \\
\hline 1883 & 5.1 & 10.2 & 1921 & 56.5 & 71.9 \\
\hline 1884 & 9.3 & 12.2 & 1922 & 71.4 & 90.1 \\
\hline 1885 & 6.3 & 5.3 & 1923 & 109.1 & 97.1 \\
\hline 1886 & 3.9 & 5.5 & 1924 & 134.1 & 102.6 \\
\hline 1887 & 8.0 & 8.0 & 1925 & 143.0 & 119.4 \\
\hline 1888 & 17.7 & 10.2 & 1926 & 197.8 & 177.8 \\
\hline 1889 & 17.5 & 28.6 & 1927 & 149.9 & 128.8 \\
\hline 1890 & 21.1 & 35.7 & 1928 & 87.4 & 115.3 \\
\hline 1891 & 17.3 & 20.0 & 1929 & 269.8 & 269.1 \\
\hline 1892 & 13.2 & 32.1 & 1930 & 253.2 & 216.2 \\
\hline 1893 & 18.0 & 25.3 & & & \\
\hline
\end{tabular}

Fuentes: elaboración propia con base en Ducoing, Inversión, 2011, y Tafunell, “Siglo”, 2011.

\section{FuENTES CONSULTADAS}

Estadísticas oficiales de comercio exterior (ECE)

Annual Statement of the Trade of the United Kingdom with Foreign Countries and Britain Possessions, Londres, Statistical Office of the Customs and Excise Department, 1856-1930. 
Anuario estadístico de la república de Chile, Chile, Oficina de Estadísticas, varios años entre 1910 y 1930.

Auswärtiger Handel des Deutschen Zollgebiets, Berlín, Reichsamt des Innern, varios años entre 1890 y 1913, y entre 1919 y 1930.

Commerce Yearbook 1926, Washington, US Department of Commerce, 1928, 2 vols.

Estadística comercial de Chile, Chile, Oficina de Estadísticas, 1840-1938.

Estadística comercial de Chile, 1897, Chile, Imprenta y Litografía Universo, 1898.

Memoria del superintendente de aduanas sobre la renta y el comercio exterior en 1901, Valparaíso, Imprenta del Universo de Guillermo Helfmann, 1902.

Resumen del comercio exterior de Chile en el año de 1907. Importación y exportación, Chile, Superintendencia de Aduanas-Departamento de Estadística Comercial/ Sociedad Imprenta y Litografía Universo, 1908.

Statistik des Deutschen Reiches. Auswärtiger Handel, Berlín, Kaiserlichen Statistischen Amt, varios años entre 1890 y 1930.

Statistik des Deutschen Reiches. Der Auswärtige Handel Deutschlands, Berlín, Statistichen Reichsmat, varios años entre 1890 y 1930.

Statistik des Deutschen Zollgebiets, Berlín, Kaiserlichen Statistischen Amt, varios años entre 1890 y 1930.

Tableau annuel du commerce avec les pays étrangers, Bruselas, Ministère des Finances, varios años entre 1890 y 1930.

The Foreign Commerce and Navigation of the United States, Washington, Bureau of StatisticsUS Department of Commerce, varios años entre 1870 y 1930.

\section{Bibliografía}

Carmagnani, Marcello, Sviluppo industriale e sottosviluppo economico: il caso cileno (1860-1920), Turín, Fondazione Luigi Einaudi, 1971, 269 pp.

CARreras, ANNA y MARC BADIA-Miró, "La fiabilidad de la asignación geográfica en las estadísticas del comercio exterior: América Latina y El Caribe (1908-1930)", Revista de Historia Económica. Journal of Iberian and Latin American History, Universidad Carlos III de Madrid, año 26, núm. 3, 2008, Madrid, pp. 355-373.

Carter, Susan B. et al. (eds.), Historical Statistics of the United States: Earliest Times to the Present (Millennial Edition), Nueva York, Cambridge University Press, 2005, 5 vols.

Censo industrial y comercial año 1937, Santiago de Chile, Imprenta y Litografía Universo, 1939.

Corporación de Fomento de la Producción, Cuentas nacionales de Chile: 19401954, Santiago de Chile, Editorial del Pacífico, 1957.

DuCOING, CRISTIÁN, "Inversión en maquinaria, productividad del capital y crecimiento económico”, tesis doctoral, Barcelona, Universitat de Barcelona, 2012. 
"Capital Formation in Machinery and Industrialization. Chile, 18441938”, ponencia presentada en el V Sound Economic History Workshop, Lund, Suecia, 2010.

"Inversión en maquinaria y crecimiento económico en el largo plazo. Chile, 1890-2005” en Recercat. Dipósit de la Recerca de Catalunya, España, Universitat Pompeu Fabra, 2010, en <http://hdl.handle.net/2072/168395>. [Consulta: 5 de septiembre de 2011.]

Federico, Giovanni y Antonio Tena, "On the Accuracy of Foreign Trade Statistics (1909-1935): Morgernstern Revisited”, Explorations in Economic History, Elsevier, vol. 28, núm. 3, 1991, pp. 259-273.

Feinstein, Charles H., National Income, Expenditure, and Output of the United Kingdom, 1855-1965, Cambridge, Cambridge University Press, 1972.

"National Statistics, 1760-1920" en Charles H. Feinstein y Sidney PoLLARD (eds.), Studies in Capital Formation in the United Kingdom, 1750-1920, Oxford, Clarendon, 1988, pp. 257-471.

Folchi, Mauricio y MARÍA del MAR Rubio, "El consumo aparente de energía fósil en los países latinoamericanos en 1925: una propuesta metodológica a partir de las estadísticas de comercio exterior” en MARÍA DEL MAR Rubio y Reto E. BERTONI (eds.), Energía y desarrollo en el largo siglo XX. Uruguay en el marco latinoamericano, Barcelona y Montevideo, Universitat Pompeu Fabra/Universidad de la República, 2008, pp. 19-71.

HARDACH, GERD, La primera guerra mundial, 1914-1918, Barcelona, Crítica, 1986.

Herrmann, Alberto, Observaciones a la estadística del comercio exterior de Chile (18891890). Calculado al cambio de 38 peniques por peso, Santiago de Chile, Sociedad Nacional de Minería/Imprenta Nacional de Moneda, 1892.

KIrsch, Henry W., Industrial Development in a Traditional Society: The Conflict of Entrepreneurship and Modernization in Chile, Gainesville, University of Florida Press, 1977.

Lagos, Ricardo, La industria en Chile: antecedentes estructurales, Santiago, Universidad de Chile, 1966.

OCDE, Medición del capital: manual de la Organización para la Cooperación y el Desarrollo Económicos 2009, Francia, OCDE Publishing, 2a. ed., 2009, 208 pp.

ORTEGA, LuIS, Chile en ruta al capitalismo: cambio, euforia y depresión, 1850-1880, Santiago de Chile, Lom Ediciones/Dirección de Bibliotecas, Archivos y Museos/Centro de Investigaciones Diego Barros Arana, 2005.

Pinto, Aníbal, Antecedentes sobre el desarrollo de la economía chilena, Santiago de Chile, Editorial del Pacífico, 1954.

Reynolds, Clark W. y Markos Mamalakis, Essays on the Chilean Economy, Illinois, Richard D. Irwin, Inc., 1965.

SAlazAR, Gabriel, Mercaderes, empresarios y capitalistas (Chile, siglo XIX), Santiago de Chile, Sudamericana, 2009.

Stevenson, David, 1914-1918. The History of the First World War, Londres, Allen Lane, 2004. 
Tafunell, Xavier, "Capital Formation in Machinery in Latin America, 1890-1930", The Journal of Economic History, Cambridge University Press, vol. 69, núm. 4, 2009, Cambridge, pp. 928-950. "La inversión en equipo de transporte de América Latina, 1890-1930: una estimación basada en la demanda de importaciones", Investigaciones de Historia Económica, Elsevier, vol. 5, núm. 14, 2009, pp. 39-67. , "Un siglo de formación de capital en América Latina (1856-1950). Ensayo de cuantificación general”, ponencia presentada en el X Congreso Internacional de la Asociación Española de Historia Económica, Carmona, septiembre de 2011. Tena, Antonio, Las estadísticas históricas del comercio internacional: fiabilidad y comparabilidad, España, Banco de España, 1992.

Tena, Antonio y Giovanni Federico, "What do We Know About the International Export Performance of the Americas between 1820 and 1940?”, sesión 15, ponencia presentada en el X Congreso Internacional de la Asociación Española de Historia Económica, Carmona, septiembre de 2011.

Wrigley, Chris (ed.), The First World War and the International Economy, Cheltenham, Edward Elgar, 2000. 